\title{
Malocclusion in Mouth-Breathing Children Caused by Nasal Obstruction
}

\author{
Cynthia M. Anggraini ${ }^{1}$, Sarworini B. Budiardjo ${ }^{2 *}$, Darmawan B. Setyanto ${ }^{2}$, Ike S. Indiarti ${ }^{2}$ \\ ${ }^{1}$ Pediatric Residency Program, Faculty of Dentistry, Universitas Indonesia \\ ${ }^{2}$ Department of Pediatric Dentistry, Faculty of Dentistry, Universitas Indonesia \\ *Email: sarworinibagio@yahoo.com
}

\begin{abstract}
This pilot study analyzed the relationship between habitual mouth breathing and malocclusion in patients diagnosed with upper airway obstruction. A crosssectional descriptive study was conducted by consecutive sampling of children/adolescents with allergic rhinitis, rhinosinusitis, and adenoid hypertrophy. Following a subject questionnaire about mode of breathing, an impression was taken, and a Vernier caliper was used to analyze the occlusion. Several types of malocclusion were found in mouth-breathing subjects. Habitual mouth breathing with an upper airway obstruction may contribute to the development of malocclusion. Ear, nose, and throat (ENT) specialists should consider treating all mouth-breathing children, regardless of etiological factors, since malocclusion risks cannot be calculated based on routinely used criteria alone.
\end{abstract}

Keywords: nasal obstruction, mouth breathing, malocclusion, child, adolescent

\section{Introduction}

Humans are designed to breathe through the nose, but, for a variety of reasons, a switch may be made to mouth breathing, with potentially serious consequences for the entire body as well as for the development of the dental and maxillary apparatus [1]. Habitual mouth breathing usually occurs as result of the need to adapt due to a blockage in the nose caused by a partial nasopharyngeal (upper airway) obstruction. The obstructive causes of mouth breathing include rhinosinusitis, nasal polyp, allergic rhinitis, tonsil and adenoid hypertrophy, and obstructive sleep apnea syndrome (OSAS), either singly or in combination [2]. Habitual mouth breathing is known as one of the many factors that can have a significant impact on overall dentocraniofacial development [3]. However, the dental literature shows conflicting results with respect to respiratory disorders and malocclusion occurrence. Some studies have found habitual mouth breathing to be the primary factor in the etiology of malocclusion and other facial abnormalities [4,5]. Others, however, have disagreed $[6,7]$.

There has been very little research conducted in Indonesia into the relationship between upper airway obstruction and malocclusion, while many international studies have failed to relate the occurrence of malocclusion to the time span from the onset of habitual mouth breathing. Therefore, this pilot study was conducted to evaluate the influence of habitual mouth breathing caused by upper airway obstruction on malocclusion prevalence. This study will provide information to aid in proposing suitable dental prevention programs and therapeutic approaches to reduce possible 
orofacial deformities related to upper airway obstruction; it will also operate as a pilot study, providing the basis of further research in the future.

\section{Method}

Approved by the Faculty of Dentistry, University of Indonesia Ethical Committee (Jakarta, Indonesia), this pilot study is a cross-sectional, descriptive study evaluating the relationship between habitual mouth breathing caused by upper airway obstruction and the time span of habitual mouth breathing on the prevalence of malocclusion in 7- to 18-year-old children. It was conducted using a consecutive sampling method on children/adolescents with allergic rhinitis, rhinosinusitis, nasal polyp, adenoid hypertrophy, and/or OSAS attending the Pediatric Respirology and Immunology Allergy Outpatient Clinic at the Kiara Maternal and Child Health Center, Cipto Mangunkusumo Hospital, Jakarta during September and October 2016. Excluded from this study were children with craniofacial deformities and syndromes, children who had had a nonnutritive sucking habit or other mouth habit for more than 3 years, children who had undergone or were in orthodontic treatment, very ill children, and those who did not consent to take part in the research. Also, no subjects had dental caries that affected the integrity of the mesiodistal diameter.

A questionnaire-based study was used to determine the subjects to be excluded and to interview the children (whether orthodontic treatment was/had been applied, whether there were complaints of disturbed nose breathing) and their parents (data on the child's breathing patterns, breast feeding and its duration, pacifier use and its duration, thumb sucking or other habits and their duration, and bottle feeding and its duration).

Informed consent was obtained from the parents or guardians of those subjects willing to participate in the study. The procedure of data collection, benefits of the research, confidentiality of data collected, and risks and discomfort of the procedure were all carefully explained to the children and their parents or guardians. Voluntary participation and withdrawal from the study at any time without prejudice were also assured.

Besides the questionnaire, a clinical evaluation and specific tests (mirror and water tests) were performed by a single examiner. The clinical evaluation involved determination of the presence/absence of the following signs: long face, drooping eyes, dark circles under the eyes, thin upper lip, dry lips, hypotonic lips, inverted lower lip, narrow nostrils, high-arched palate, and inadequate lip seal. A mirror test was conducted, which involved placing a two-sided mirror below the child's nostrils and observing the formation of vapor condensation stemming from their respiration. Fogging on the upper part of the mirror indicated nasal breathing, while fogging on the lower part or both parts indicated mouth breathing. A water test was performed after the mirror test. For this test, the child held a small amount of water in their mouth and kept the lips together (in contact) without swallowing for 3 minutes, during which time the effort of the lip commissure was observed. Children who were unable to maintain the lips in full contact for three minutes were considered mouth breathers [8].

Intraoral orthodontic examination was performed first with the mouth open and later with the teeth in occlusion using examination gloves and two mouth mirrors. 
After intra-oral examination, impressions were made in elastomer impression material with fabricated trays for all participants. The impressions were rinsed under running water and disinfected with diluted sodium hypochlorite solution before casting. The inter-arch occlusion dental classification was identified based on the method of Barnett as described previously.9 The measurements of overjet and overbite were performed using Vernier calipers with a precision of $\pm 0.1 \mathrm{~mm}$. All data were collected by one investigator.

\section{Results}

This study involved seven patients (one female, six male): two aged 7, one aged 12 , three aged 14, and one aged 16 years. Regarding the etiology of habitual mouth breathing, there were five patients diagnosed with allergic rhinitis, one with rhinosinusitis, one with adenoid hypertrophy, and none with nasal polyps or OSAS (Fig. 1). Among the patients, three had no malocclusion, two of them had class 2 division 1 malocclusion, one had anterior open bite malocclusion, and the other had class 2 division 1 combined with posterior crossbite (Fig. 2).

There were three patients with a 1-2-year history of mouth breathing, one with a 2-3-year history, and three with a mouth-breathing history of more than 3 years (Fig. 3). No malocclusion was found in three patients (43\%), all of them with a 12 -year history of mouth breathing. Anterior open bite was found in the one patient (14\%) who had a 2-3-year history of mouth breathing. Class II division 1 malocclusion $(29 \%)$ was found in two of the patients with a greater than 3 -year history of mouth breathing, while the other one (14\%) had class II division 1 malocclusion combined with posterior crossbite (Table 1).

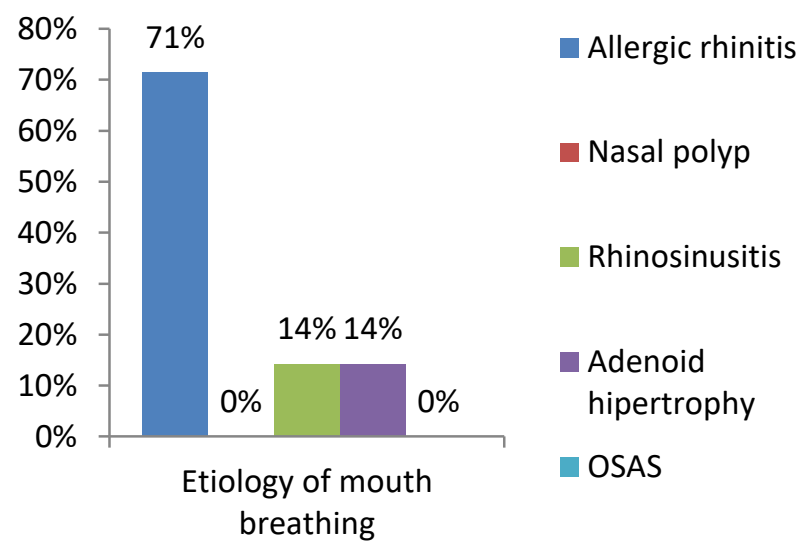

Fig. 1 Distribution of habitual mouth breathing by etiology. 


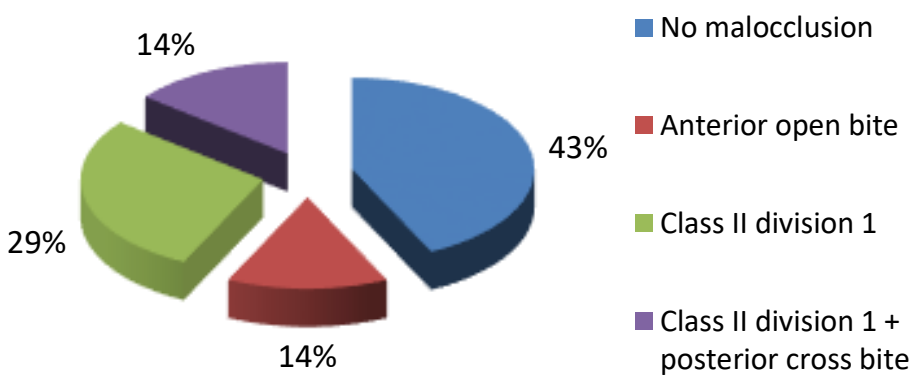

Fig. 2 Distribution of malocclusion in mouth-breathing patients.

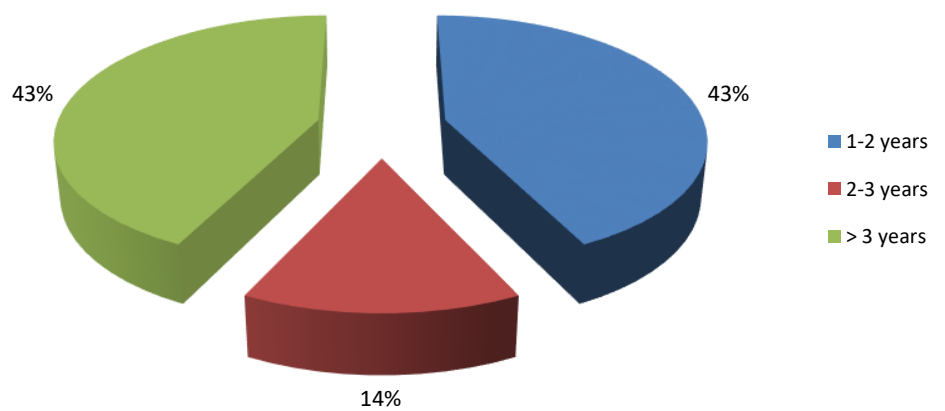

Fig 3. Distribution of mouth-breathing patients by duration of mouth breathing.

\section{Discussion}

This study was conducted to evaluate the occlusal characteristics of mouth-breathing patients with upper airway obstruction. The dental inter-arch relatiohship in the three planes of space is the basic clinical parameter in understanding patient occlusion and its behavior when exposed to unbalanced muscular activities. Generally, the triad of class II malocclusion, anterior open bite, and posterior crossbite has been described as the occlusal pattern for mouth-breathers $[4,9]$.

Of the seven mouth-breathing patients in this study, four (57\%) had malocclusions, which comprised class II division 1, anterior open bite, and posterior crossbite. This finding was in accordance with those of other studies [4,9]. These types of malocclusion may occur due to an altered respiratory pattern as the result of 
mouth breathing and lead to postural alterations, such as open lips, lowered or anterior tongue position, and posterior inferior rotation of the mandible in order to stabilize the airway.

Postural alterations in soft tissues have been noted to change the equilibrium of the pressure exerted on teeth and facial bones, thus altering these structures. 4 When tongue posture is normal, the tongue rests on the hard palate. As the tongue is displaced downward, the support for the lingual surfaces of the maxillary molars is lost, as is the counterbalance for the buccinator muscles. Flaccid lips decrease the labial support of the maxillary anterior teeth. These muscle or soft-tissue imbalances and postural relationships may result in a posterior crossbite, an anterior open bite, and/or protrusion of the maxillary incisors [10].

Another point which must be considered is the time span between the initiation of mouth breathing and the malocclusion outcome. This research showed that patients with a 1-2-year history of mouth breathing did not have malocclusions, while those with 2-3 years or more of mouth breathing showed specific types of malocclusion. From this finding, we can theorize that children with greater obstruction and/or a longer history of mouth breathing could develop more malocclusion than children with less severity and a shorter mouth breathing history. However, further research is required to confirm this and to investigate the relationships between malocclusion types and time.

The findings of this study suggest that, from the orthodontic point of view, ear, nose, and throat (ENT) specialists should consider treating all mouth-breathing children, regardless of the etiological factors, since it is not possible to identify the risk of developing malocclusion based solely on routinely used criteria.

\section{Conclusion}

From the results of the present study, it can be concluded that habitual mouth breathing among children is associated with specific types of malocclusion, including class II division 1, anterior open bite, and/or posterior crossbite. The temporal history of mouth breathing, especially durations of more than 3 years, may influence malocclusion establishment.

\section{References}

1. O'Hehir, TE, Francis A. Mouth and nasal breathing. Hygiene and Prevention: Profile in Oral Health [Internet]; 2012. Available from: http://www.bronsonfamilydentistry.com/upload/Mouth and Nasal Breathing.pdf.

2. Cuc A, Cuc O. Mouth breathing-an etiological factor of dental and maxillary abnormalities. Analele Universității din Oradea, Fascicula: Ecotoxicologie, Zootehnie şi Tehnologii de Industrie Alimentară. 2013;12(B):49-57.

3. Kaur R, Chandrashekhar G, Mody DR. An insight into relationship of hypertrophied adenoids \& tonsils and dentofacial form. J Dental Med Sci. 2014;13(9):48-54.

4. Osiatuma VI, Otuyemi OD, Kolawole KA, Ogunbanjo BO, Amusa YB. Occlusal characteristics of children with hypertrophied adenoids in Nigeria. Int Orthod. 2015 ;13(1):26-42.

5. Bezzo, SM. The effect of oral respiration on the dental occlusion in patients with respiratory tract allergies. JBCD. 2005;17(2):87-92. 
6. Coelho AR, Tanaka O, Ribeiro JS, Machado MÂ, Camargo ES. Transverse craniofacial dimensions in Angle Class II, Division 1 malocclusion according to breathing mode. Braz Oral Res. 2010;24(1):70-5.

7. Góis EG, Ribeiro-Júnior HC, Vale MP, Paiva SM, Serra-Negra JM, Ramos-Jorge ML, Pordeus IA. Influence of nonnutritive sucking habits, breathing pattern and adenoid size on the development of malocclusion. Angle Orthod. 2008;78(4):647-54.

8. Motta LJ, Alfaya TA, Mesquita-Ferrari RA, Fernandes KP, Bussadori SK. Gender as risk factor for mouth breathing and other harmful oral habits in preschoolers. Braz J Oral Sci. 2012;11(3):377-80.

9. Souki BQ, Pimenta GB, Souki MQ, Franco LP, Becker HM, Pinto JA. Prevalence of malocclusion among mouth breathing children: do expectations meet reality?. Int J Pediatr Otorhinolaryngol. 2009;73(5):767-73.

10. Soxman JA. Upper Airway Obstruction in the Pediatric Dental Patient, Gen. Dentistry July-August. 2004:313-5. 